\title{
Correction to: Tunable room temperature ferromagnetism and optical bandgap of CdS:Er nanoparticles
}

\author{
B. Poornaprakash ${ }^{1}$ (D) Ramanadha Mangiri ${ }^{2} \cdot$ Abdulaziz A. Al-Kheraif $^{3} \cdot$ Darshan Devang Divakar $^{3} \cdot$ Y. L. Kim ${ }^{1}$. \\ Mirgender Kumar ${ }^{4} \cdot$ M. Siva Pratap Reddy ${ }^{5}$
}

Published online: 25 June 2021

(c) The Author(s), under exclusive licence to Springer-Verlag GmbH, DE part of Springer Nature 2021

\section{Correction to: Applied Physics A (2021) 127:359 https://doi.org/10.1007/s00339-021-04508-7}

In the original publication of the article, B. Poornaprakash and Y.L Kim's affiliation was incorrect. The correct affiliation appears as below.

Department of Electronic Engineering, GangneungWonju National University, Gangneung, 25457, Republic of Korea

Publisher's Note Springer Nature remains neutral with regard to jurisdictional claims in published maps and institutional affiliations.

The original article can be found online at https://doi.org/10.1007/ s00339-021-04508-7.

B. Poornaprakash, Ramanadha Mangiri are equally contributed.

Y. L. Kim

ylkim@gwnu.ac.kr

$\triangle$ Mirgender Kumar

mkshekhawat22@gmail.com

$\triangle$ M. Siva Pratap Reddy

dr.mspreddy@gmail.com

1 Department of Electronic Engineering, Gangneung-Wonju National University, Gangneung 25457, Republic of Korea

2 Department of Physics, Sri Venkateswara University, Tirupati 517502, India

3 Dental Biomaterials Research Chair, Dental Health Department, College of Applied Medical Sciences, King Saud University, P.O Box 10219, Riyadh 11433, Saudi Arabia

4 Department of Electronic Engineering, Yeungnam University, Gyeongsan 38541, South Korea

5 School of Electronics Engineering, Kyungpook National University, Daegu 41566, South Korea 